ANTONIO INTERGUGLIELMI

Studium Theologicum Galilaeae, Israel

\title{
LA PRIVACY NEL DIRITTO CANONICO E I RAPPORTI CON LE LEGISLAZIONI NAZIONALI DELLA COMUNITA' EUROPEA
}

Sommario: Premessa. - 1. Le norme canoniche sugli archivi ecclesiastici e la tutela dei dati personali. La normativa statale di tutela dei dati personali e i rapporti in Italia con la Chiesa. - 2. Il trattamento, la conservazione e la divulgazione dei dati sullo Status delle persone da parte della Chiesa. 3. La creazione di banche dati informatiche ad accesso protetto sul Web: il diritto di accesso ai propri dati personali (can. $487 \$ 2$ ) e la tutela della intimità della Persona (can. 220) in collegamento con le Direttive a tutela della privacy dell'Unione Europea. - Conclusioni.

\section{Premessa}

Il tema della tutela della riservatezza nasce già a fine ' 800 nell'ambito della common law, facendo sorgere così già prima del XX secolo nella dottrina giuridica un dibattito sull'argomento. Gli ordinamenti di diritto romano si occupano della questione dopo il secondo conflitto mondiale, sulla spinta dei diritti alla vita privata e famigliare, riconosciuti nella Convenzione Europea dei Diritti dell'uomo del 1950, ampliati molto più tardi nella Carta dei diritti fondamentali dell'Unione Europea del 2000¹.

\footnotetext{
${ }^{1}$ Il concetto di privacy si ritiene che nasca negli stati Uniti alla fine dell'800 con la pubblicazione dell'articolo The right of privacy da parte di Warren e Brandeis, un avvocato e un giudice suo amico, perché l'avvocato che riteneva che i giornali dell'epoca si fossero occupati troppo delle vicende private della moglie. In questo
} 
Con l'espansione dell'utilizzo dei media, la sempre maggiore tutela riconosciuta alla riservatezza dei dati personali, è andata progressivamente aumentando, in modo particolare negli ultimi anni con il crescente diffondersi di internet e dei social media. Si sono create nuove problematiche di tutela dei dati personali, spesso rese molto complesse dalla difficoltà di arginare un fenomeno di "trasmissione di informazioni e quindi anche di dati" in continua espansione tecnologica.

Questa problematica non può essere ignorata dall'ordinamento ecclesiale.

Vi sono due motivi fondamentali che rendono la questione centrale per l'ordinamento canonico: il primo è costituito dal fatto che la Chiesa da sempre è depositaria della memoria e della storia dei popoli, attraverso gli archivi ecclesiastici, delle diocesi, dei monasteri e anche delle singole parrocchie. Si tratta di una serie impressionante di dati personali custoditi in questi archivi che da un lato permette la ricostruzione della storia e della vita di intere generazioni, dall'altro rappresenta la vita dei singoli fedeli; è di tutta evidenza l'intreccio tra la componente storica e la componente spirituale di questi straordinari "data-base". In secondo luogo il diritto della Chiesa riconosce esplicitamente tra i suoi diritti fondamentali il rispetto della persona, tra cui rientra quello del "diritto al rispetto della buona fama e della riservatezza di ogni persona", sancito nel Codice di diritto canonico del 1983 al canone 220.

Non si tratta quindi soltanto di approfondire la questione della conciliazione tra le disposizioni previste a tutela della privacy dalla legislazione dei singoli ordinamenti e le norme previste nel diritto canonico a livello di conferenze Episcopali nazionali, quanto di cercare

articolo sostenevano un interessante parallelismo tra privacy e diritto di proprietà, rifacendosi al celebre brocardo ius escludendi alios: così come il proprietario di un fondo ha diritto che estranei non vi entrino, allo stesso modo si ha il diritto che altri non si occupino delle vicende private personali: S. D WARREN, L. D. BRANDEIS, The Right to privacy, in: Harvard Law Review, Vol. IV, December 15, 1980, n. 5. Per una disamina storica, A. LefeBvre-TeIllarD, Introduction historique au droit des personnes et de la famille, Parigi 1996. 
di adeguare l'applicazione del principio che tutela la buona fama della persona (canone 220) all'evoluzione dei sistemi di catalogazione e trasmissione dei dati all'interno del diritto ecclesiale.

Adeguare, quindi, ove è necessario, la normativa canonica alle nuove esigenze, per la gestione degli archivi ecclesiastici "correnti" in modo che sia garantita un'efficace tutela dei diritti fondamentali della persona.

Nella nostra ricerca prenderemo come riferimento particolare la normativa della Comunità Europea, analizzando a titolo di esempio la situazione della Chiesa in Italia, nella cui legislazione statale si è avuto un progressivo sviluppo ed è a tutt'oggi tra le più complete tra quelle degli Stati democratici contemporanei ${ }^{2}$ che hanno recepito le direttive Europee. Si potranno così trarre alcune indicazioni utili anche per altre situazioni nazionali³.

La tutela dei dati personali riconosciuta nella legislazione di tutti gli Stati moderni, coinvolge infatti il rapporto tra l'ordinamento della Chiesa e le norme giuridiche statali: nella legislazione di molte nazioni sono state promulgate leggi che tutelano il trattamento dei dati personali, istituendo specifiche autorità a cui vengono riconosciute forme di controllo e l'autorità di sanzionare severamente l'inosservanza delle norme.

Nel loro compito di disciplinare il trattamento dei dati cosiddetti "sensibili" delle singole Chiese, le Conferenze Episcopali nazionali dovranno quindi sempre tener conto di queste leggi statali.

\footnotetext{
${ }^{2}$ Il modello normativo della legislazione italiana si fonda sui due principi della normativa Comunitaria in materia di privacy, che quindi troviamo anche nelle leggi di altri Stati dell'UE, tra di loro confliggenti: il principio della tutela del diritto della persona, in particolare della dignità umana, e il principio della libertà del trattamento dei dati.

${ }^{3}$ La legislazione civile che per prima si è occupata in Europa della tutela del diritto alla privacy è quella inglese, che ha formulato una serie di regole per la tutela dei dati personali e delle immagini. Cfr. l'analisi di M. Jones, Privacy, North Pomfret, Vt., 1974, p. 210.
} 


\section{Le norme canoniche sugli archivi ecclesiastici e la tutela dei dati personali. La normativa statale di tutela dei dati personali e i rapporti in Italia con la Chiesa}

Nella legislazione canonica a carattere generale, il codice di diritto canonico (da ora anche CIC) del 1983, ai canoni da 482 a 490, disciplina gli Archivi Vescovili, il canone 491 la tenuta dell'Archivio delle Chiese e il Canone 535 quella dell'Archivio Parrocchiale; tali norme non accennano però all'aspetto della tutela dei dati personali, se non in collegamento a quanto previsto dal già menzionato canone 220. Ne sono infatti attuazione i canoni 487 e 488 che regolano la tenuta del cosiddetto archivio corrente, nonché il canone 535, per quanto concerne i doveri del Parroco per il suo archivio.

Nel 1997 un documento della Pontificia Commissione per i Beni Culturali della Chiesa intitolato La funzione Pastorale degli archivi ecclesiastici, accenna alla questione della tutela dei dati personali: per quanto composto in una logica di carattere pastorale, in esso si accenna alla problematica del rispetto dei dati personali contenuti negli archivi:

Esso consentirà la produzione degli altri strumenti utili alla consultazione del materiale (cataloghi, repertori, regesti, indici) e permetterà l'utilizzazione dei moderni sistemi informatici onde collegare le varie sedi archivistiche e favorire una ricerca su ampia scala. Avvalendosi delle nuove tecnologie, è inoltre opportuno conservare in un altro luogo protetto la copia dei documenti ${ }^{4}$.

È doveroso, però, che vengano posti dei limiti alla consultazione dei fascicoli personali e di altri carteggi che per natura loro sono riservati o che i responsabili riterranno tali. Non ci riferiamo all'archivio

\footnotetext{
${ }^{4}$ La versione originale è un opuscolo edito dalla Pontificia Commissione per i beni Culturali della Chiesa, Lettera circolare La funzione pastorale degli archivi ecclesiastici, 2 febbraio 1997 (Città del Vaticano 1997, p. 45). Ė stata anche pubblicato in Il Regno-Documenti 1997/15, p. 501-506 e in Enchiridion Vaticanum 16/119-169.
} 
segreto della curia diocesana, di cui espressamente trattano i cann. 489-490, ma in generale agli archivi ecclesiastici ${ }^{5}$.

Fino agli anni novanta, in cui si è avuta una serie di normative sulla tutela dei dati personali, in parallelo alla sempre più crescente espansione della forma di comunicazioni tramite internet, la Chiesa si preoccupava di garantire soltanto la conservazione dei dati negli archivi ecclesiastici, a partire da quelli parrocchiali e delle Curie, sia quali strumenti di memoria storica, sia come forma di testimonianza dell'opera ecclesiale.

In Italia, come in molti altri paesi Europei, la legislazione statale già si era adeguata a garantire una tutela maggiore dei dati "sensibili"6: in Italia, con la legge n. 675 del 1996, poi assorbita dal d.lgs. 196 del 2003, era stata introdotta nell'ordinamento giuridico statale la prima normativa relativa alla tutela delle persone e di altri soggetti per il rispetto dei loro diritti nel trattamento dei dati personali: la Chiesa Italiana, che come noto tratta istituzionalmente, da sempre, i dati personali, si è trovata nella necessità di introdurre delle norme di adeguamento nel proprio ordinamento giuridico ${ }^{7}$.

Sollecitati da questa normativa si giunse da parte della Chiesa Italiana alle disposizioni della Conferenza Episcopale italiana (nel seguito "CEI") del 20 ottobre 1999: un decreto generale intitolato Disposizioni per la tutela del diritto alla buona fama e riservatezza. Le norme contenute in questo decreto costituiscono un'attuazione e un'interpretazione in chiave pratica di quanto previsto dal canone 220

\footnotetext{
${ }^{5}$ Pontificia Commissione per i beni culturali della Chiesa, La Funzione pastorale degli archivi ecclesiastici, 2 febbraio 1997.

${ }^{6} \mathrm{Si}$ intende con questo termine i dati che possono rivelare l'origine razziale ed etnica, le convinzioni religiose, filosofiche o di altro genere, le opinioni politiche, l'adesione a partiti, sindacati, associazioni od organizzazioni a carattere religioso, filosofico, politico o sindacale, lo stato di salute e la vita sessuale.

${ }^{7}$ Cfr. R. Bотта, Trattamento dei dati personali e confessioni religiose (dalla legge 31.12.1996, n. 675 al d.lgs 21.5.1999 n. 135), Diritto Ecclesiastico 1(1999), p. 882 ss.

${ }^{8}$ Questo decreto riceve la necessaria recognitio della Congregazione dei Vescovi, con decreto 4 ottobre 1999, prot. 960/83.
} 
del CIC '83. In qualche modo sono anche un "ponte" con la normativa civile dello Stato italiano.

Il decreto della CEI già in premessa riconosce che "è opportuno dare più articolata regolamentazione al diritto della persona alla buona fama e alla riservatezza riconosciuto dal can. 220 del codice di diritto canonico", ribadendo nel contempo il diritto nativo e proprio della Chiesa cattolica di "acquisire, conservare e utilizzare per i suoi fini istituzionali i dati relativi alle persone dei fedeli, agli enti ecclesiastici e alle aggregazioni ecclesiali".

Alcune disposizioni di questo decreto sono utili da sottolineare per quanto diremo più avanti.

Vi si trova una distinzione fondamentale: da una parte i Registri, dall'altra gli elenchi e gli schedari:

a. Registri sono i libri in cui è annotata, in successione cronologica, l'avvenuta celebrazione dei sacramenti e altri fatti di natura e rilevanza ecclesiale.

Questi dati riguardano dunque lo Status delle persone e la loro appartenenza ecclesiale.

Vi rientrano pertanto i dati che vengono estrapolati da: libro dei battesimi, libro dei defunti, Status animarum, registro delle cresime, delle sacre ordinazioni e tutti gli altri previsti dal diritto particolare che contengano dati sullo status delle persone (can. $535 \$ 1$ ).

Questi Registri sono principalmente quello diocesano e quello dell'archivio parrocchiale.

Il trattamento di dati attinenti le attività istituzionali rientra dunque nella disciplina di carattere speciale; viceversa, il trattamento di dati afferenti fornitori e dipendenti va condotto secondo la disciplina di carattere generale, e dunque è soggetto a tutti gli adempimenti della legge ordinaria in ordine alla tutela della privacy, al pari di qualunque altro ente non ecclesiastico.

Quanto al trattamento di dati afferenti attività di tipo solidaristico ed umanitario, la normativa speciale potrà trovare applicazione se la Parrocchia si limiti a raccogliere e gestire esclusivamente i dati 
afferenti la componente missionaria e catechetica dell'attività complessivamente svolta.

Ciò vuol dire che i dati raccolti debbono strettamente essere funzionali alle finalità costitutive e legittimanti della Confessione cattolica: la promozione dell'individuo e della sfera collettiva, l'accoglienza, l'ascolto e l'orientamento, l'evangelizzazione, l'attività sacramentale. Si tratta, in buona sostanza, di attività che di per sé non abbisognano, ordinariamente, dell'acquisizione di dati personali i quali, se raccolti, restano nell'alveo di applicazione della normativa speciale.

Sotto questo aspetto negli ultimi anni sono state numerose le questioni concernenti il diritto al cosiddetto "sbattezzo", cioè alla cancellazione dei richiedenti dai registri dei battezzati: la maggior parte delle richieste ha avuto come spunto i diritti al trattamento dei dati della ricordata legge 675 del 1996.

Ricordiamo in proposito soltanto che il Garante per la protezione dei dati personali $i^{9}$ ha affermato che "il registro del battesimo in riferimento ad una persona che si dichiara atea, non contiene dati trattati illecitamente, né notizie inesatte o incomplete, ma documenta un fatto realmente avvenuto". Pertanto le richieste di "sbattezzo" sono state soddisfatte con speciale annotazioni, nel Registro dei battesimi, della defectio a fide dell'interessato.

b. Schedari ed elenchi, sono invece gli strumenti con cui i soggetti raccolgono i dati per lo svolgimento delle diverse attività istituzionali che appartengono all'ordinamento canonico.

E' interessante rilevare che questo decreto della Conferenza Episcopale Italiana del 1999 riceve nella disciplina dell'ordinamento giuridico statale un tacito riconoscimento con l'aggiunta di altre indicazioni anch'esse utili per la migliore configurazione di una disciplina canonica di protezione della privacy.

\footnotetext{
${ }^{9}$ Il Garante è un'autorità amministrativa indipendente imposta a livello europeo da molti trattati, a cominciare da quello di Schengen sulla libera circolazione delle persone.
} 
Ci riferiamo all'art. 26, comma 3 del citato D. Lgs. 30 giugno 2003, n. 196, Codice in materia di protezione dei dati personali. Detta norma stabilisce una deroga al comma 1 dello stesso articolo che prevede " $i l$ consenso scritto dell' interessato per il trattamento dei dati sensibili...".

Il comma 3 dell'articolo 26 dispone infatti che "il comma 1 non si applica al trattamento:dei dati relativi agli aderenti alle confessioni religiose e ai soggetti che con riferimento a finalità di natura esclusivamente religiosa hanno contatti regolari con le medesime confessioni, effettuato dai relativi organi, ovvero da enti civilmente riconosciuti, sempre che idati non siano diffusi o comunicati fuori delle medesime confessioni.

Queste ultime determinano idonee garanzie relativamente ai trattamenti effettuati, nel rispetto dei principi indicati al riguardo con autorizzazione del Garante; (Omissis...)".

Senza entrare nelle specificità della legislazione italiana che qui non interessano, come la figura e il ruolo del Garante ${ }^{10}$ della Privacy, due elementi ci indicano che cosa la legislazione statale può in generale richiedere alla Chiesa per la tutela dei dati personali dei cittadini:

a. I soggetti di cui si trattano i dati devono avere contatti regolari con la confessione, per finalità di natura esclusivamente religiosa;

b. Il trattamento va fatto dagli Organi delle confessioni o da Enti ecclesiastici civilmente riconosciuti;

c. I dati non vanno diffusi o comunicati fuori delle medesime confessioni.

Quest'ultima disposizione ci interessa in particolare.

La ratio della norma appare questa: la tenuta dei dati negli Archivi della Curia o di quelli parrocchiali, così come disposto dal can. 535 $\$ 1$ (battesimi, matrimoni, defunti e altri stabiliti dalle Conferenze Episcopali o dai Vescovi diocesani), hanno una finalità collegata allo

\footnotetext{
${ }^{10}$ Autorità cd. “Tutoria”, ora disciplinata dal d.lgs. n. 196/2003, Parte III, Titolo II, artt. 153 ss., che in Italia si è più volte pronunciata -come vedremo più avanti - sulla tutela dei dati religiosi sensibili.
} 
status del fedele, al cui interesse sono autorizzati, oltre alla persona, le autorità della singola confessione: Vescovo, Parroci, ma non gli altri singoli fedeli.

Ci troviamo dunque dinanzi a due interessi confliggenti: la comunicazione dei dati alle persone effettivamente interessate e autorizzate, e la tutela della riservatezza di questi dati, sicuramente sensibili, perché concernono lo "status" della persona.

A livello generale l'ordinamento canonico garantisce già questa protezione: in primis attraverso il riconoscimento del diritto alla riservatezza quale diritto di ogni uomo, che deriva dal diritto naturale (canone 220 del $\mathrm{CIC}^{11}$ ), e poi dando disposizioni di protezione dei dati personali dei fedeli posseduti negli archivi, con i canoni 487 e 488, che regolano l'accesso agli archivi (correnti, no storico, né quello segreto) della Curia, e il canone 535, per la protezione dei dati conservati nei libri dello stato delle persone della Parrocchia.

Da queste norme possiamo dunque desumere una serie importante di indicazioni pratiche:

a. solo gli interessati possono richiedere copia o certificati circa il loro status di fedele;

b. a norma del canone 487, gli interessati vantano un vero e proprio diritto soggettivo a ricevere la certificazione: ius est iis quorum interest;

c. i documenti sui quali si ha questo diritto devono riguardare lo stato della propria persona e non essere coperti da segreto.

In alcuni Stati sono sorte alcune questioni circa le dichiarazioni da rilasciare relativamente ai dati conservati negli archivi ecclesiastici: accenniamo qui soltanto alle questioni che sono nate nello Stato Italiano relativamente al certificato di battesimo per i figli adottivi: nel 1984 vi è stato anche un intervento della Conferenza Episcopale Italiana (delibera n. 18 del 6 settembre 1984). Si tratta di una questione

\footnotetext{
${ }^{11}$ Circa il contenuto di questo canone cfr. D. Mogavero, Diritto alla buona fama e alla riservatezza dei dati personali, Ius Ecclesiae 12(2000), p. 589 ss., e P. SKonieczny, Koncepcja teoretycznoprawna ochrony dobrego imienia w kan. 220 Kodeksu Prawa Kanonicznego Jana Pawła II, Annales Canonici 4(2008), pp. 271-285.
} 
piuttosto articolata di rapporti tra ordinamento canonico e normativa di diritto statale sulla famiglia, che non possiamo qui approfondire ${ }^{12}$.

\section{Il trattamento, la conservazione e la divulgazione dei dati sullo Status delle persone da parte della Chiesa}

Nelle leggi degli Stati Europei le norme sulla tutela dei dati personali hanno avuto una notevole evoluzione dagli anni '90, determinando l'adozione di forme sempre più decise di tutela dei dati cosiddetti "sensibili", talvolta prevedendo anche strutture di controllo e di disciplina nei confronti degli Organismi che gestiscono dati personali.

Si tratta in qualche modo del riconoscimento normativo e della codificazione di nuovi diritti, emersi quindi alcuni anni dopo la pubblicazione del CIC del 1983, ma non per questo estranei ai suoi canoni.

Nel rapporto con questa normativa statale in continua evoluzione, l'ordinamento canonico solo apparentemente è rimasto fermo alle norme del 1983. In realtà esso si era fatto portatore ben prima dell'ordinamento statale della finalità di trattare i dati personali dei fedeli contenuti nei propri archivi con particolare attenzione al rispetto della persona.

Se assicurare la tutela della buona fama della persona e della riservatezza (can. 220) non è dunque una novità per l'ordinamento canonico $^{13}$, allo stesso tempo, però, la Chiesa ha dovuto difendere e ribadire il proprio diritto a tenere l'archivio e i documenti sullo stato giuridico canonico dei suoi fedeli, in forza della propria autonomia.

In questo senso la bipartizione, a cui accennavamo nel paragrafo precedente, adottata dal decreto della CEI del 1999 - tra registri ed archivi da un lato e elenchi/schedari dall'altro - può servirci da criterio guida.

Se il diritto di conservare i registri da parte della Chiesa deve essere contemperato con il diritto della persona al rispetto della sua

\footnotetext{
${ }^{12}$ Sul punto si può approfondire in C. Scognamiglio, Sul diritto dell'adottato ad ignorare l'identità dei propri genitori naturali, in: Giurisprudenza italiana, I, 2, 1988.

${ }^{13}$ Sul contenuto della nuova tutela alla riservatezza contenuta nel CIC del $1983 \mathrm{si}$ veda ad esempio, V. Marcozzi, II diritto alla propria intimità nel nuovo Codice di diritto canonico, La Civiltà Cattolica 134/IV (1983), pp. 573-580.
} 
riservatezza, si dovranno adottare misure "efficaci" per garantire ad esempio per i dati custoditi nelle Curie - questa tutela. Non si potrà dunque consentire l'accesso a questi registri (correnti) se non attraverso personale qualificato, al fine di rilasciare la certificazione ai diretti interessati. E la stessa cosa deve avvenire per i Registri Parrocchiali, per i quali la funzione e la custodia è affidata al Parroco, ma su cui vigila sempre l'autorità del Vescovo.

Questa tutela nel trattamento dei dati, in attuazione sempre del principio riconosciuto al canone 220 del CIC - scrive in dottrina Castillo Lara - spetta non solo al fedele in quanto incorporato a Cristo mediante il Battesimo, ma anche all'uomo non credente e non battezzato.

In qualche modo la Chiesa è stata antesignana rispetto alla legislazione dello Stato, perché ancor prima che fossero riconosciute le protezioni dei dati personali attraverso le leggi civili, l'ordinamento canonico già esigeva al suo interno il diritto al rispetto della riservatezza e dell'intimità della persona: diritto che deriva dal diritto naturale e che quindi deve essere riconosciuto a chiunque ${ }^{14}$.

Per il diritto della Chiesa sarebbe dunque un errore legare l'esigenza della tutela dei dati della persona con la custodia dei dati dei fedeli al solo diritto di evitare che altri ne abbiano conoscenza; la tutela che occorre assicurare è ancora più ampia e si sostanzia nel rispetto della dignità della persona. Riguarda infatti tutti quegli uomini che non desiderano far conoscere le loro scelte personali, quindi anche in forma negativa: non voler far sapere ad esempio che un padre, pur cristiano, non ha voluto far battezzare i propri figli.

Per queste ragioni il diritto all'intimità, ormai riconosciuto nella maggioranza degli ordinamenti civili degli Stati democratici, all'interno della comunità ecclesiale riceve una tutela ancora più decisa: perché la sicurezza della custodia dei dati contenuti negli archivi ecclesiastici è l'attuazione di uno dei diritti fondamentali dell'uomo.

\footnotetext{
${ }^{14}$ R. Castillo Lara, I doveri e i diritti dei Christeifideles, Salesianum XLVIII, 1986.
} 
Nel codice di diritto canonico vi è anche la possibilità di un risarcimento dovuto da chi non assolve a questa funzione di custodia dei Registri a cui è tenuto: fino a poter configurare, nei casi di maggiore gravità, il delitto previsto dal canone $1390 \$ 2$ "lesione della buona fama" causata da un dovere d'ufficio non rispettato.

Dovere di conservazione ed esigenza di tutela della riservatezza dei dati contenuti nei Registri vanno rispettati con ogni accortezza: se per il Parroco si tratterà di non permettere a chiunque in Parrocchia di accedere ai Registri, nella Curia si dovranno formulare delle regole per disciplinare la consultazione degli stessi Registri da parte del solo personale autorizzato.

\section{La creazione di banche dati informatiche ad accesso protetto sul Web: il diritto di accesso ai propri dati personali \\ (can. $487 \$ 2$ ) e la tutela della intimità della Persona (can. 220) in collegamento con le Direttive a tutela della privacy dell'Unione Europea}

Come abbiamo visto la legislazione italiana consente alla Chiesa cattolica e agli enti ecclesiastici civilmente riconosciuti che il trattamento dei dati, raccolti con riferimento a finalità di natura esclusivamente religiosa, si svolga secondo le modalità e le garanzie previste dal diritto canonico (art. 26 del cd Codice della Privacy ${ }^{15}$ ), purché rispettino l'ordinamento statuale.

Nel codice di diritto canonico non si esclude la possibilità della costituzione di una banca dati informatizzata; ma questa è consentita accanto ad un archivio cartaceo, che potrà essere sostituito integralmente: non potrà dunque mai mancare la versione cartacea, ufficiale, relativamente ai registri obbligatori.

Ma questi dati possono essere resi accessibili tramite internet, seppure solo ad alcune persone selezionate, sotto protezione di un codice di accesso (password)? Partiamo da un primo dato tecnico: numerosi studi sull'argomento dimostrano che il grado di sicurezza

${ }^{15}$ D.Lgs. 30 giugno 2003, n. 196, cit. 
di questi sistemi, soprattutto se non si dispone di tecnologie adeguate, è molto basso.

E necessario precisare che le "misure di sicurezza" vengono sempre descritte nelle leggi sulla privacy statali come misure di protezione idonee a prevenire il rischio di una perdita o distruzione dei dati anche solo accidentale, "di un accesso non autorizzato o di un trattamento non consentito o non conforme alle finalità della raccolta", confermando la definizione di "sicurezza" verso la quale sembrano orientate le legislazioni di tutti i Paesi che si sono già occupati del problema.

Un ostacolo nell'adozione di queste banche dati è anche di carattere tecnico: ove fosse ammissibile per il diritto canonico, la Curia sarebbe in grado di realizzare una banca dati non meramente locale che tuttavia disponga di un sufficiente grado di protezione, tale da non infrangere innanzitutto le norme dello Stato sulla privacy?

La Comunità Europea, ad esempio, ha adottato nella riunione del Consiglio 20 febbraio 1995, in vista della adozione della direttiva sul trattamento dei dati personali, una norma che prevede che gli Stati membri dispongano, nelle rispettive legislazioni, per un diritto al risarcimento del danno "per chiunque subisca nocumento da un trattamento illecito di dati personali o dalla violazione delle norme che disciplinano le banche-dati"16; nell'ordinamento italiano quindi non adottare "adeguate misure di protezione" configura un fatto illecito ${ }^{17}$.

${ }^{16}$ Cfr. Direttiva 95/46/CE del Parlamento Europeo e del Consiglio del 24 ottobre 1995, relativa alla tutela delle persone fisiche con riguardo al trattamento dei dati personali, nonché della libera circolazione di tali dati. Questa direttiva costituisce l'atto legislativo fondamentale in materia di protezione dei dati personali nell'Unione europea; vi si stabiliscono le condizioni generali di liceità del trattamento di dati personali e i correlativi diritti che spettano ai soggetti interessati. Sempre in questa direttiva è prevista la creazione di autorità di controllo indipendenti nazionali. In virtù di queste norme, una persona deve dare il proprio consenso esplicito ed essere informata prima che i suoi dati personali siano trattati. Cfr. F. Pizzetri, Privacye il diritto europeo alla protezione dei dati personali. Dalla Direttiva 95/46 al nuovo Regolamento europeo, Torino 2016.

${ }^{17}$ La legge italiana, la ricordata 675 , equipara il trattamento dei dati, ai fini della risarcibilità del danno, all'esercizio di attività pericolose che determinano, a carico 
Questo per un esempio legato ai problemi che possono facilmente sorgere in ambito civile dall'adozione di una banca dati sul web; ovviamente l'indagine andrà calibrata sulle singole legislazioni nazionali.

Il rispetto per la vita privata e familiare è stato inserito tra i diritti fondamentali nella Carta fondamentali, firmata a Nizza nel dicembre del 2000, riconfermata a Strasburgo nel 2007 e poi riconosciuta come documento avente valore giuridico, successivamente al Trattato di Lisbona del 13 dicembre $2007^{18}$.

Tuttavia, poiché non tutti gli Stati membri hanno attuato uniformemente questa normativa e per il fatto che il rapido progresso tecnologico ha mutato le modalità di raccolta, di consultazione e di utilizzo dei dati personali, la Commissione Europea, il 25 gennaio 2012, ha presentato un nuovo pacchetto legislativo inteso a riformare la normativa dell'Unione Europea sulla protezione dei dati ${ }^{19}$. Dopo tre anni di lavoro sono state approvate le nuove norme, pubblicate ad aprile del 2016, che entreranno in vigore a maggio del $2018^{20}$.

di chi le esercita, una presunzione di responsabilità a norma dell'art. 2050 del codice civile. Questo comporta che la legge presume la colpa del gestore della banca di dati e - invertendo l'onere della prova - pone a suo carico ogni possibile conseguenza dei danni cagionati a terzi, se egli non prova di avere adottato tutte le misure idonee ad evitare il danno.

${ }^{18}$ Cfr. AA. VV. La Carta dei diritti dell'Unione Europea. Casi e materiali, (a cura di G. Bisogni-G. Bronzini-V. Piccone), 2010.

${ }^{19}$ Il pacchetto si apre con una comunicazione strategica sui principali obiettivi politici della riforma, cui fa seguito una proposta di regolamento generale per modernizzare i principi sanciti dalla direttiva del 1995 sulla protezione dei dati personali e una nuova formulazione sulla tutela del trattamento dei dati personali nell'ambito della cooperazione di polizia e giudiziaria in materia penale. A conferma della complessità della materia, soltanto a fine 2015 il Parlamento Europeo (a livello di commissione) e il Consiglio (a livello di ambasciatori) hanno raggiunto un accordo sulle nuove norme in materia di protezione dei dati, dunque dopo quasi tre anni di lunghe trattative

${ }^{20}$ Si tratta del regolamento (UE) n. 2016/679, del Parlamento europeo e del Consiglio, del 27 aprile 2016, relativo alla protezione delle persone fisiche con riguardo al trattamento dei dati personali, nonché alla libera circolazione di tali dati e che abroga la direttiva 95/46/CE (regolamento generale sulla protezione dei dati) e 
Il discorso della tutela dei dati a livello Europeo deve ovviamente contemperare anche la necessità di garantire la sicurezza: le norme del 2016 andranno pertanto coordinarsi con l'accordo quadro raggiunto con gli Stati Uniti, relativamente allo scambio di informazioni e alla protezione dei dati, noto come l'Accordo quadro, il cui scopo è di assicurare un elevato grado di protezione dei dati personali trasferiti nel quadro della cooperazione transatlantica nella lotta al terrorismo e alla criminalità organizzata ${ }^{21}$.

La prima legge Italiana sulla privacy, la n. 675/1996, dava una definizione di banca dati: "Qualsiasi complesso di dati personali, ripartito in una o più unità dislocate in uno o più siti, organizzato secondo una pluralità di criteri determinati tali da facilitarne il trattamento"; il decreto CEI del 1999 contiene alcune disposizioni sugli archivi informatici, ma non affronta la questione dei dati inseriti in banche dati, non locali, accessibili via web.

Lo scopo della Banca dati è ovviamente quello di rendere i dati immediatamente fruibili, al fine anche di consentire un facile accesso agli stessi a chi ne ha diritto. Sotto questo aspetto le Banche dati informatizzate vanno create e favorite, anche all'interno della Chiesa.

Tuttavia, come sopra ricordato, il diritto di accesso ai dati contenuti nei Registri, secondo quanto previsto dal decreto CEI del 1999 e dalla più generale normativa canonica, spetta soltanto al titolare dei dati medesimi.

la direttiva (UE) 2016/680 del Parlamento europeo e del Consiglio, del 27 aprile 2016, relativa alla protezione delle persone fisiche con riguardo al trattamento dei dati personali da parte delle autorità competenti a fini di prevenzione, indagine, accertamento e perseguimento di reati o esecuzione di sanzioni penali, nonché alla libera circolazione di tali dati, e che abroga la decisione quadro 2008/977/GAI del Consiglio.

${ }^{21}$ Cfr. David H. Fla herty, Protecting privacy in surveillance societies: the Federal Republic of Germany, Sweden, France, Canada, and the United States, Londra 1989, che scrive a p. 12: "The problem of controlling surveillance is rife with ambivalences. governments are the worst offenders against privacy, at least in terms of demonstrable abused against individuals and groups...". 
In altri termini, hanno diritto d'accesso soltanto le persone il cui interesse è motivato dal fatto che il documento concerne lo statum suae personae: questo, specifica il codice, soltanto se il documento per sua natura è pubblico (non coperto da segreto).

L’ammissibilità di queste banche dati via web nell'ordinamento ecclesiale ci pone dinanzi all'esigenza di contemperare due interessi: da un lato si trova il diritto di accesso ai dati personali degli aventi diritto $^{22}$, dall'altra la tutela dei dati stessi, in applicazione prima di tutto dei principi del canone 220. Si tratta di un impegno che riguarda non solo i provvedimenti che dovranno adottare le Conferenze Episcopali dei singoli Stati, ma anche le disposizioni amministrative del Vescovo della Chiesa particolare ${ }^{23}$.

Dall'inserimento sul web della banca dati non c'è infatti alcun vantaggio per il singolo per facilitare la fruizione dei propri dati: infatti se l'accesso venisse consentito ad un numero elevato di persone, si perderebbe la sicurezza circa la riservatezza dell'archivio. Il singolo dovrà sempre rivolgersi a chi è autorizzato a consultare i Registri, siano essi cartacei o informatizzati (can. $487 \$ 1$ ): nella Curia, Vescovo, Moderatore o Cancelliere, in parrocchia il proprio Parroco o persona di fiducia da lui delegata.

In questo modo cade la principale ragione sostenibile che potrebbe giustificare la costituzione di queste banche dati sul web: facilitare il diritto d'accesso ai propri dati. Allo stesso tempo, non può essere sufficiente ad adottare la banca dati via web, né la possibilità di ogni parroco di accedere ai dati di un'altra parrocchia o dell'intera diocesi, né la maggiore facilità di comunicazione dei dati tra Diocesi e Parrocchie.

\footnotetext{
${ }^{22} \mathrm{G}$. Finocchiaro, Difficile convivenza nella pubblica amministrazione tra diritto di accesso e tutela dei dati personali, in: Guida alla tutela della privacy, Il Sole-24 ore, Guida al Diritto, 1997, pp.100-108.

${ }^{23}$ Un impegno dunque da armonizzare con le singole legislazioni nazionali che recepiscono però le citate disposizioni della CEE. L'impegno è notevole: cfr. ad esempio per la Spagna J. Otaduy, La Iglesia católica ante la Ley española de Protección de Datos, Ius canonicum XLV 90 (2005), pp. 529-555.
} 
Il Codice prescrive infatti che il Parroco ha il dovere della custodia (can. 486, $535 \$ 1$ ), dell'aggiornamento ed è responsabile del rilascio dei certificati: l'inserimento dei dati in un sistema informatico accessibile via web, per quanto limitato e controllato da chiavi di accesso appare comunque poco prudente e dunque non accettabile: renderebbe anche superfluo tale dovere di custodia e aggiornamento dei dati, che potrebbero diventare di fatto accessibili via web anche a persone non autorizzate esplicitamente dal Parroco, ancorché venute in possesso della password per accedere alle informazioni $\mathrm{i}^{24}$.

Tra l'altro, il disposto del can. 487, "Nemini licet illud ingredi nisi de Episcopi aut Moderatoris curiae simul et cancellarii licentia", indica il Vescovo e il Cancelliere e altri operatori della Curia diocesana autorizzati per dovere d'ufficio come coloro che devono avere esclusivamente la possibilità di accedere all'archivio: indirettamente si afferma che essi devono essere "proprietari" esclusivi delle informazioni e messi in grado di disporne.

Il can. 487, che tratta dell'Archivio comune di Curia, dove sono conservati e ordinati gli atti curiali (cf can. $482 \$ 1$ ), «gli strumenti e le scritture che riguardano le questioni spirituali e temporali della diocesi» (can. $486 \$ 2$ ), regolamenta chiaramente le modalità di protezione e l'accesso all'Archivio corrente.

Non riguarda, quindi, come precisa il can. $489 \$ 1$, l’Archivio segreto di Curia, accessibile solo al vescovo e contenente documenti particolarmente riservati, né l'Archivio storico (can. $491 \$ 2$ ), dove sono conservati documenti di valore storico, ma che non hanno più riferimento a persone ancora viventi e ai quali possono avere accesso gli studiosi.

Neppure si può interpretare che l'Archivio cosiddetto "corrente", inaccessibile alle persone non autorizzate, e la cui chiave deve essere

\footnotetext{
24 "In generale si può dire che i libri parrocchiali rispondono ad esigenze sia anagrafiche sia pastorali, e consentono al parroco, che ne è personalmente responsabile, di disporre di strumenti conoscitivi della realtà parrocchiale assai utili per l'esercizio del suo sacro ministero", così O. PASQuINELLI, I lineamenti della disciplina canonica sugli archivi ecclesiastici, Quaderni di diritto ecclesiale 7 (1994), p. 373.
} 
custodita dal Vescovo e dal Cancelliere, potrebbe prevedere un accesso singolo, attraverso una password di volta in volta viene creata e affidata al Parroco che deve consultare: si tratta di un espediente talmente complesso e macchinoso che, senza considerare comunque il permanere di profili di sicurezza non risolti, non se ne vede la ragione per adottarlo.

Circa il diritto di accesso ai dati, inoltre, il canone 487, al $₫ 2$, peraltro, non riconosce un vero e proprio diritto di accedere neppure per consultare documenti che riguardano la propria persona; c'è solo il diritto dell'interessato, di avere copia autentica di quegli atti che per loro natura sono pubblici.

E' pacifico in dottrina che tali norme intendano tutelare il diritto di ogni persona alla buona fama e alla riservatezza, in applicazione del più volte citato canone 220, di cui costituisce applicazione pratica: «Non è lecito ad alcuno ledere illegittimamente la buona fama di cui uno gode, o violare il diritto di ogni persona a difendere la propria intimità» ${ }^{25}$.

Riteniamo inoltre che non garantisce sufficientemente il rispetto della riservatezza la protezione tramite password dei dati contenuti nell'archivio: mentre per consultare i dati personali di un archivio cartaceo si ha sempre bisogno dell'intermediazione di una persona, Vescovo o Cancelliere, Parroco o suo delegato, una semplice chiave "elettronica" espone i dati alla possibilità che persone non autorizzate magari in forma fortuita - acquisiscano la possibilità di venire in possesso del codice di accesso.

L'Archivio deve essere chiuso (can. $486 \$ 2$ "diligenter clausa") e la sua chiave custodita dal vescovo e dal cancelliere. Il permesso di ingresso di altre persone (che non siano i collaboratori di Curia

\footnotetext{
${ }^{25}$ Cfr. A. Solferino, I diritti fondamentali del fedele: il diritto alla buona fama ed alla intimità, in: Diritto per valori ed ordinamento costituzionale della Chiesa, a cura di R. Bertolino, Torino, 1996, p. 375 e J. KRukowski, Prawa wiernych do dobrej opinii i do własnej intymności. Komentarz do kan, 220, Prawo Kanoniczne 39(1996), pp. 227-236.
} 
autorizzati) deve essere regolato e la facoltà di avere certificati o copie deve avvenire per legittima richiesta (ca. $487 \$ 2)^{26}$.

A queste ragioni esposte che si aggiungono dei possibili profili di problematicità con il diritto statale che potrebbero creare queste banche dati: i dati relativi alla confessione religiosa in tutti gli ordinamenti che hanno adottato una normativa sulla protezione dei dati personali, rientrano tra i "dati sensibili" (in Italia nel d.lgs 196/2003 art. 4, lettera d), perché sono ritenuti tra i dati idonei a rivelare le convinzioni religiose: questi dati in molte delle leggi Statali emanate a tutela dei dati personali, vengono assimilati a quelli circa lo stato di salute e la vita sessuale. Quindi trattati con molto rigore.

Occorre quindi prendere in considerazione che l'eventuale violazione nel trattamento dei dati di religione da parte della Chiesa, che sono dati sensibili per lo Stato, comporta sempre il rischio di una doppia rivendicazione da parte della persona che si ritiene danneggiata nel suo diritto: non solo quindi in sede di giustizia ecclesiale, ma anche in ambito statale e civilistico.

In questo senso è interessante il caso della catechista svedese Bodil Lindqvist, che ha portato ad una Sentenza della Corte di Giustizia della Comunità Europea, del 6 novembre 2003: la signora, catechista nella parrocchia di Alseda (Svezia), aveva creato alcune pagine internet per consentire ai parrocchiani che si preparavano alla cresima di avere informazioni utili. Queste informazioni, oltre a nome e cognomi, fornivano vari elementi, sia sulla situazione familiare che sui loro interessi. Condannata nel suo paese, ella presentò un ricorso alla Corte di giustizia Europea contiene tra le altre un interessante orientamento: spetta all'ordinamento interno dei singoli paesi garantire con norme proprie il giusto equilibrio tra diritti e interessi in gioco.

In dottrina tra i canonisti tuttavia è aperta la discussione sulla necessità di un'armonizzazione tra la necessità di tutela dei diritti all'intimità e riservatezza del singolo con lo spirito di comunione che deve animare la comunità ecclesiale: si è talvolta affermato che

\footnotetext{
${ }^{26}$ Il Regolamento o statuto di Curia può prevedere come procedere per le richieste e rilascio di documenti e copie depositati nell'Archivio.
} 
i diritti dei fedeli non devono essere concepiti come delle pretese individualistiche, contrapposte allo spirito di comunione e al principio gerarchico ${ }^{27}$.

Se è condivisibile l'opinione che ritiene necessario approfondire l'attuazione pratica del principio generale del can. 220, dall'altra il principio contenuto in questa norma rimane comunque il dato di riferimento di tutti i possibili sviluppi. Le norme del codice di diritto canonico necessitano di una riflessione non tanto sull'aspetto della tutela della protezione dei dati delle persone, quanto semmai di un adeguamento per regolamentare le nuove forme di catalogazione e di trasmissione dei dati attraverso le nuove tecnologie: si stanno addirittura già pensando delle applicazioni per gli smartphone dove raccogliere velocemente e dovunque i dati da inserire poi nei registri della parrocchia, e cose simili ${ }^{28}$.

Occorre dunque un'integrazione della normativa canonica in attesa della quale occorre agire con estrema prudenza, guidati dai principi generali a cui abbiamo fatto riferimento, con lo studio attento delle norme dello Stato in cui ci si trova dedicate all'argomento. Per l'Italia, cui abbiamo fatto riferimento, il Garante della Privacy ha per esempio chiesto che le confessioni religiose determinino nel loro ordinamento idonee garanzie per la tutela dei dati personali dei loro fedeli, adeguandole alle nuove esigenze di comunicazion $e^{29}$.

Sarà opportuno adottare una regolamentazione delle banche dati, da parte delle Conferenze Episcopali e anche nelle singole diocesi, che tenga conto di quanto detto sopra. E' di fondamentale importanza

\footnotetext{
${ }^{27}$ Così C.J. Errázuriz M. Sul rapporto tra comunione e diritto nella Chiesa, Fidelium Iura 4/1994, p. 51.

${ }^{28}$ A. Vitalone, Buona fama e riservatezza in diritto canonico, Ius Ecclesiae 14(2002), p. 278-279, nelle conclusioni. dove, relativamente alla situazione della chiesa italiana afferma che " $i l$ Decreto generale della C.E.I. ha risposto a molti dubbi che la normativa a tutela della riservatezza poteva suscitare anteriormente alla sua promulgazione, ma altri, come si è tentato di mostrare, sono rimasti insoluti".

${ }^{29}$ Si trova nell'autorizzazione del Garante del 19 giugno 2008, nr. 3: "Le confessioni adottino idonee garanzie relativamente ai trattamenti effettuati nel rispetto dei principi indicati con l'autorizzazione in parola".
} 
che vengano stabilite l'opportunità e i limiti di queste banche dati, escludendo l'ammissibilità delle banche dati via web.

Per le banche dati consentite, eminentemente di carattere locale, devono comunque essere aggiornate costantemente alla luce delle generali norme di sicurezza ed è indispensabile assicurarsi che in ogni caso vengano adottate misure che riducano al minimo sia il pericolo di perdita o distruzione dei dati, sia di illecita intromissione nei sistemi da parte di soggetti non autorizzati.

\section{Conclusioni}

La problematica della tutela della privacy e dei dati personali esige anche da parte della normativa canonica un ulteriore approfondimento, anche in considerazione del sempre più diffuso uso delle comunicazioni attraverso la rete internet da parte delle diocesi e delle parrocchie, che introducono così dati sensibili dei fedeli, esponendosi ad una violazione della normativa che i singoli Stati hanno adottato per la tutela della privacy.

Anche se abbiamo limitato il nostro studio alla normativa della Comunità Europea, che fornisce le linee direttive per la legislazione da attuare nei singoli Stati, per quanto concerne l'utilizzo della rete internet, riteniamo necessario che le Conferenze Episcopali Nazionali, nonché le singole Diocesi, si debbano adeguare per garantire la tutela dei molti dati sensibili che, in ragione dell'attività di religione e culto, si trovano a gestire ${ }^{30}$.

Un esempio è dato dalla crescente richiesta che viene da diverse Diocesi di costituire archivi informatici in rete. In questo ambito riteniamo che non sussistano ragioni sufficientemente valide per giustificare l'adozione di archivi informatici generali dei certificati

\footnotetext{
30 "Otwarcie sie jednej osoby wobec drugiej w sprawach dotyczacych jej intymności nie może być wymuszone przez kogokolwiek, także przez osoby sprawujące władze $w$ Kościele, np. przełożonego zakonnego lub biskupa diecezjalnego", così scrive J. KRUKowski in Prawa wiernych do dobrej opinii i do własnej intymności. Komentarz do kan, 220, cit ., p. 236. Anzi il Vescovo dovrà vigilare sugli Enti ecclesiastici della sua diocesi perché sia garantita la tutela di questi dati.
} 
sullo status delle persone attraverso sistemi di archiviazione e trasmissione dei dati via web, anche qualora si adottino sistemi di sicurezza e criptografia dei dati ${ }^{31}$, perché, a quanto previsto nel codice di diritto canonico, questa forma di divulgazione dei dati contenuti nei Registri è in contrasto con le norme del CIC, non solo perché si espongono i dati ad una possibile illecito utilizzo, ma anche perché viene meno il ruolo dei garanti e custodi dei dati, come previsto nel CIC medesimo.

Attraverso internet si può ammettere pertanto soltanto l'inoltro, tramite posta elettronica, di documenti a coloro a cui è riconosciuto il diritto di ricevere copia della certificazione, ex canone $487 \$ 2$ : del resto già da tempo questa forma di comunicazione, mirata all'interessato, poteva avvenire tramite il fax ${ }^{32}$.

L'adozione di un sistema di gestione web-based con i dati sacramentali parrocchiali informatizzati e accessibili da tutte le Parrocchie, espone alla violazione del canone 220 circa la riservatezza di molti dati che in Curia sono conservati.

\footnotetext{
${ }^{31}$ In questo senso anche M. Mosconi, Internet e la produzione e comunicazione di documenti canonici: un legame da approfondire, Quaderni di diritto ecclesiale 14(2001), pp. 370-371, dove relativamente agli archivi, scrive: "Per quanto riguarda il rilascio di attestazioni relative a dati di archivio si noti che occorre procedere per via cartacea tradizionale. Per quanto riguarda la parrocchia è stabilito, ad esempio, che devono es sere tenuti i libri parrocchiali (non sostituibili da supporto informati co) e i relativi attestati sullo stato canonico dei fedeli o gli atti che possono avere rilevanza giuridica (cf can.535) e che questi devono essere sottoscritti dal parroco o dal delegato e muniti di sigillo. L'utilizzo di internet, anche a questo livello, è fruttuoso per svolgere un ruolo ausiliario, consentendo le comunicazioni tra i diversi archivi: per la comunicazione di dati da un registro parrocchiale a un altro (cf can. 895 per la confermazione, can. 1054 per l'ordine, can. 1122 per il matrimonio: la possibilità di usare la posta elettronica è espressamente prevista dalla normativa CEI) o per altre trasmissioni di informazioni (il can. $470 \$ 3$ del CIC 1917, ad esempio, prescriveva l'annuale trasmissione dei dati dei registri parrocchiali alla curia: il diritto partico lare può ancora prevedere tale richiesta)".

${ }^{32}$ Dello stesso avviso M. Mosconi, op. ult. Cit., p. 370: "Mediante internet è invece possibile inoltrare per posta elettronica i documenti a coloro ai quali si desidera darne conoscenza o ai quali compete il vero e proprio diritto di richiedere copia (cfcan. $487 \$ 2$ )".
} 
Allo stesso tempo un sistema di archiviazione e consultazione online, confligge con il can. 487 e il can. 535: i responsabili dell'Archivio di Curia e dell'Archivio Parrocchiale non potrebbero più svolgere il ruolo di garanti e custodi della sicurezza dei dati contenuti nei propri Registri. Si tratta di un dovere da espletare maxima cura e in riferimento a documenta omnia (can. $486 \$ 1$ ).

Un sistema che prevede l'accesso alle informazioni tramite password, oltre ai rilievi sulla difficoltà nel valutare la validità delle forme di protezione, nonché alla complessità ad adottare strumenti tecnici di accesso realmente efficaci e quindi costosi, non assolve a quanto richiesto dal codice: la necessità della previa autorizzazione del Vescovo o del Moderatore di Curia insieme al Cancelliere per entrare a consultare i dati ivi contenuti, il dovere della custodia e della protezione dei dati contenuti nei Registri Parrocchiali, da parte del Parroco (can. $535 \$ 4)^{33}$.

L'adozione del sistema della password, seppure riservata a poche persone, configura un'autorizzazione data una volta per tutte all'accesso dei dati: anche se in linea di principio limitata alle persone in possesso della chiave di accesso (appunto la password) si tratterebbe però di una autorizzazione a carattere generale, in violazione di quanto richiesto dal Codice.

33 "Gli Archivi di Curia infatti, dovendo conservare tutta la documentazione, contengono informazioni su persone che non sono di pubblico dominio e che per questo non devono essere conosciute né divulgate. Per questo il canone che stiamo commentando non riconosce un diritto di accedere neppure per consultare documenti che riguardano la propria persona; c'è solo il diritto, secondo il paragrafo $2^{\circ}$, di ottenere da parte di chi è interessato, copia autentica di quegli atti che per loro natura sono pubblici. La norma intende tutelare il diritto di ogni persona alla buona fama e alla riservatezza: "Non è lecito ad alcuno ledere illegittimamente la buona fama di cui uno gode, o violare il diritto di ogni persona a difendere la propria intimità» (can. 220)", così G. Trevisan, Le chiavi dell'archivio di Curia (can. 487), Quaderni di diritto ecclesiale 8 (1995), p. 113. Della stessa opinione, M. Mosconi, Internet e la produzione e comunicazione di documenti.., cit., p. 370: "Lo strumento di internet può consentire infine una facile diffusione dei documenti in formato informatico, anche se le problematiche concernenti la sicurezza sconsigliano l'offerta di archivi costantemente accessibili sulla rete." 
L'attenzione che l'ordinamento canonico pone per il rispetto dell'intimità della persona (canone 220) trova attuazione anche attraverso la disposizione del canone $491 \$ 1$. Questa norma estende la competenza del Vescovo diocesano anche agli archivi delle chiese cattedrali, collegiate, parrocchiali e delle altre chiese presenti nella sua diocesi: si prevede che il Vescovo abbia cura dei relativi atti e documenti, assicurandosi della loro diligente conservazione. Ma con l'adozione di una banca dati via web, anche questo canone perderebbe di efficacia pratica.

La questione va seguita con attenzione: la Chiesa da sempre si è mostrata nel suo ordinamento come modello da seguire per promuovere e rispettare i diritti legati alla dignità naturale dell'uomo, tra cui rientra quello del rispetto dell'intimità della persona ${ }^{34}$.

Il controllo sull'osservanza di questi principi di garanzia dell'intimità della persona - ora riconosciuti anche dalle legislazioni statali - garantisce la Chiesa da ingerenze indebite dello Stato: l'affermazione della propria libertà e della legittima autonomia va infatti difesa dimostrandosi credibile nell'osservanza delle norme canoniche, prima di tutto, che molto spesso assorbono e applicano poi anche quelle civili, come deve avvenire in questo caso.

Diversamente - in questo aspetto che riguarda il rispetto della persona, ma anche in altri ambiti, come per quello economico e amministrativo in cui i rapporti con la legislazione dello Stato sono molteplici - si legittimerà un intervento sempre maggiore da parte dell'autorità civile nella vita stessa della Chiesa, che dovrà e potrà stabilire limiti e imporre regole all'interno della attività ecclesiale.

\footnotetext{
${ }^{34}$ J. KRU Kowski, Prawa wiernych do dobrej opinii i do własnej intymności. Komentarz do kan, 220, cit., ricorda come già Papa Pio XII sottolineava come qualsiasi autorità sociale dovesse assicurare il rispetto dell'intimità della persona: "Intymność każdej osoby ludzkiej jest jednym z tych dóbr duchowych, do którego prawo wynika z samej natury ludzkiej. Dlatego powinno być chronione przez każda władzę społeczna. Magisterium Kościoła od dawna było zainteresowane ta ochrona. Już papież Pius XII w jednym ze swych przemówień, wygłoszonym do uczestników międzynarodowego kongresu psychologii stosowanej, wskazał na istotę tej intymności i potrzebę jej poszanowania”.
} 


\section{Privacy in Canon Law and the relations with national laws of the European Community}

With the progressive use of computer systems and with the growing use of media, especially in recent years with the growing spread of internet and social media, the issue of personal data protection has created new needs that require adjustment of canonical regulation.

The new issues concerning protection, often made very complex by the difficulty of stifling a phenomenon of "information transmission and therefore also of data" in continuous technological expansion, have made necessary to adapt the rules about privacy protection, to the European Community rules and to the individual countries rules that have to get them and that we will deal with them in our study.

Church, which has always been the custodian of memory and history of peoples, through ecclesiastical archives, dioceses, monasteries and even each parish, is required therefore to ensure the protection of the data in its possession, which represent the life of the its churchgoers.

In addition, among its fundamental rights, the law of the Church has always recognized the respect of the person, including "the right to the respect of good reputation and confidentiality of each person", which is enshrined in the Code of Canon Law of 1983 in canon 220.

Besides, personal data protection involves the connection between the Church's legal order and the laws of the States: in the legislation of many nations, laws that protect the processing of personal data have been promulgated; those rules have to be kept by the National Bishops' Conferences to regulate and adapt the canonical regulation on the treatment of the so-called "sensitive" data to them.

Parole Chiave: privacy; dati personali; riservatezza; buona fama; archivi; Curia; Europa; Internet; dati; web based

KEY WORDs: privacy; personal data; confidentiality; good reputation; archives; Curia; Europe; Internet; data; web based

\section{Nota o Autorze:}

Prof. Antonio Interguglielmi - sacerdote della diocesi di Roma, professore di diritto canonico dello Studium Theologicum Galilaeae di Israele, affiliato alla Facoltà di Teologia della Pontificia Università Lateranense di Roma, ha pubblicato nel 2012 un libro nella Collana giuridica della LEV, 
i Decreti singolari, dedicato all'attività amministrativa della Diocesi, e diversi articoli soprattutto di diritto canonico amministrativo e penale. E' Direttore dell'Ufficio per le Aggregazioni Laicali e le Confraternite della Diocesi di Roma e amministratore di diversi Enti ecclesiastici e Fondazioni della stessa Diocesi. 